\title{
Hybrid Clustering and Routing Algorithm for Node Performance Improvement in Wireless Sensor Networks
}

\section{Gopal Ram}

M.Tech. Student,

Department of CSE

Kautilya Institute of Technology \& Engineering

Jaipur, Rajasthan (India)

E-Mail: gopalram02sept1993@gmail.com

\section{Chetan Kumar}

Associate Professor, Department of CSE

Kautilya Institute of Technology \& Engineering

Jaipur, Rajasthan (India)

E-Mail: chetanmnit@yahoo.co.in

Article History: Received: 10 November 2020; Revised 12 January 2021 Accepted: 27 January 2021; Published online: 5 April 2021

\begin{abstract}
These days Wireless Sensor Networks (WSN) have been used in various Internet of Things (IoT) applications viz., healthcare monitoring, disaster management, smart buildings, smart farming etc. it is one of the substitutes for solving distinct problems of IoT in various areas. This paper proposed a hybrid clustering and routing algorithm. We develop a simulation area for node movement and communication. A hybrid clustering and routing (HCR) protocol proposed for reliable and efficient data collection in large-scale wireless sensor network. Theoretical analysis and simulation results will prove the connectivity and efficiency of the network topology generated by HCR.
\end{abstract}

Index Terms-Wireless sensor network, joint cluster, routing, throughput, energy, node life time.

\section{INRODUCTION}

WSN is an association of compact micro sensors with wireless communication capabilities. Like many advance technologies, WSN owe its root in heavy industrial applications as well as military applications. The first wireless network that is inline with the latest WSN is the Sound Surveillance System (SOSUS) developed on submerged acoustic sensors. Sensors in SOSUS were distributed in the Pacific ocean Atlantic oceans.

Stimulated by the developments pertaining to Internet in 1960s and 1970s to develop the hardware for today's Internet, Defence Advanced Research Projects Agency (DARPA) initiated the Network (DSN) program in 1980[21]. The motive was to explore the design challenges related to WSN. With the birth of DSN and its penetration into education through Carnegie Mellon University and the Massachusetts Institute of Technology, WSN technology could find its base in household, education and civilian scientific research.

Very soon, public and private communities started deploying sensors to monitor air quality, detect forest fire, forecast weather, prevent natural disaster etc. The sensors however at that time were bulky, expensive and made use of proprietary protocols. The use of such WSNs thus 
weighed down the industry which used it. This disproportionate relation of high cost with low volume of sensors declined their pervasive use.

Realizing the potential of the network, industry and academia joined hands to solve the engineering challenges associated with sensors and lead to the production of modern sensors: low cost miniature size sensors, having simplified development and maintenance tasks.

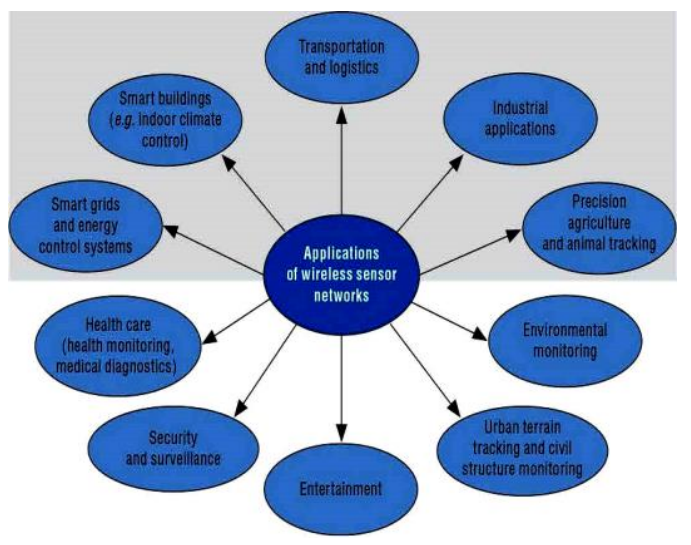

Figure 1: Wireless Sensor Networks Applications

The most frequently used wireless sensor networks applications in the field of Transport systems such as monitoring of traffic, dynamic routing management, and monitoring of parking lots, etc., use these networks.

Rapid emergency response, industrial process monitoring, automated building climate control, ecosystem and habitat monitoring, civil structural health monitoring, etc., use these networks.

In order to gather data more efficiently, a clustering hierarchy algorithm is used for data communication in wireless sensor networks (WSNs). This algorithm is one of the major techniques to improve the energy efficiency in WSNs and it provides an effective manner to maximize the lifetime of WSNs. Hierarchical protocols based on clustering hierarchy are proposed to save energy of WSNs in which the nodes with higher remaining energy could be used to collect data and transmit it to a base station. However, most of the previous approaches based on clustering hierarchy have not considered the redundant data collected by the adjacent nodes or nodes overlap each other.

\section{Proposed Methodology AND SySTEM MOdeL}

Let consider simulation area 500 meter X 500 Meter. The following flow chart represents the work flow- 


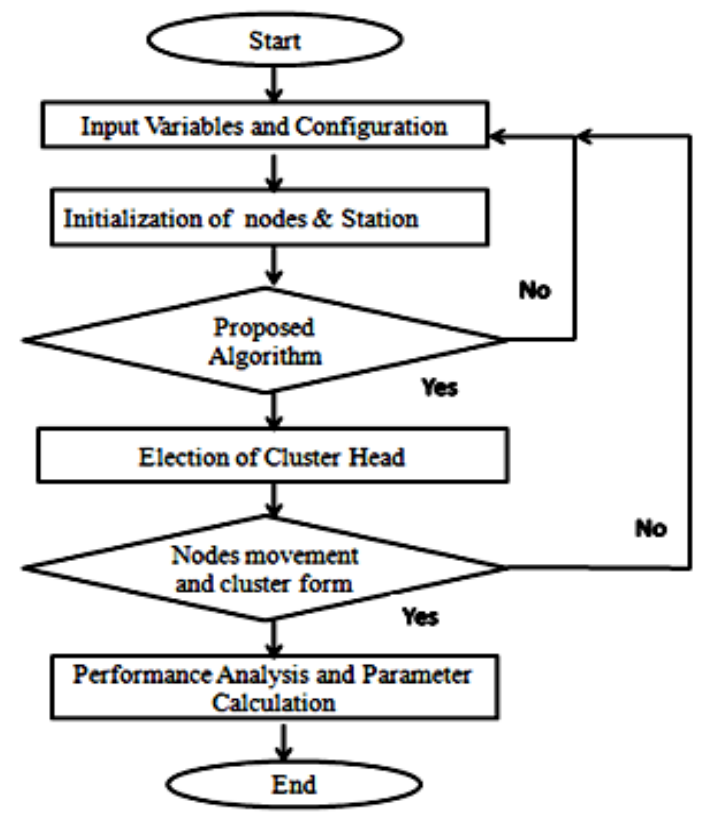

Figure 2: Flow chart

\subsection{Energy Efficient Hierarchical Clustering}

Energy Efficient Hierarchical Clustering (EEHC) is a randomized, distributed clustering algorithm that prolongs the network lifetime. In this part sensor nodes are organized into clusters with a hierarchy of cluster heads. The $\mathrm{CHs}$ collects the information from the nodes within their clusters and send the aggregated data to the BS through the hierarchy. This algorithm is based on two stage clustering: single-level and multi-level clustering. At the single-level clustering stage, each sensor node becomes a $\mathrm{CH}$ on the basis of a pre-defined probability $\mathrm{p}$ and announces itself as the volunteer $\mathrm{CH}$ to its neighbors within khops distance. Any node which receives this announcement becomes member of the closest cluster. If a node does not hear any announcement within a preset time interval $\mathrm{t}$, then it will become a forced $\mathrm{CH}$. This time interval $t$ is calculated on the basis of the duration for a packet to reach a node that is k-hops away. The energy consumed for sending the information to the sink depends on the parameters $\mathrm{p}$ and $\mathrm{k}$.

At the second stage, same process is applied from bottom-up to multilevel clustering. Assume there are $\mathrm{h}$ levels in a clustering hierarchy, among which level- 1 being the lowest one and level$\mathrm{h}$ being the highest one. Then level-1aggregates the data from its cluster members and send it to level-2 CHs, and so on. Finally the level-h CHs send the aggregated data to the base station. The cost of transmitting the information to the base station is the power consumed by sensor nodes to send data to level-1, then energy used by level-1 CHs to the base station via $\mathrm{h}-$ hop $\mathrm{CHs}$ at different hierarchical levels.

\subsection{Modified low energy adaptive clustering hirerchy}

That leads us to start thinking about constructing the networks. To further improve energy efficiently, two approaches introduced in the papers are summarized in the following:

LEACH is a cluster-based wireless sensor networking protocol. LEACH adapts the clustering concept to distribute the energy among the sensor nodes in the network. LEACH improves the 
energy-efficiency of wireless sensor networking beyond the normal clustering architecture. As a result, we can extend the life time of our network, and this is the very important issue that is considered in the wireless sensor networking field.

In LEACH protocol, wireless sensor networking nodes divide themselves to be many local clusters. In each local cluster, there is one node that acts as the base station (or we can call it "cluster-head"). Hence, every node in that local cluster will send the data to the cluster-head in each local cluster. The important technique that makes LEACH be different from the normal cluster architecture (the drain the nodes battery very quickly) is that LEACH uses the randomize technique to select the cluster-head depending on the energy left of the node.

\section{SIMULATION RESULT}

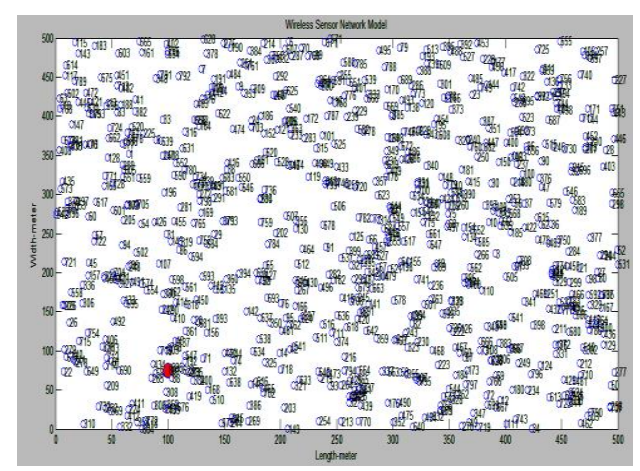

Figure 3: Simulation area 500m X 500m

Figure 3 show the simulation scenario where length and width of wireless sensors network area is $500 \mathrm{~m} X 500 \mathrm{~m}$. Total nodes taken are 100 to 1000 . In this step all variables, mobile agent and configuration are initialize the simulation.

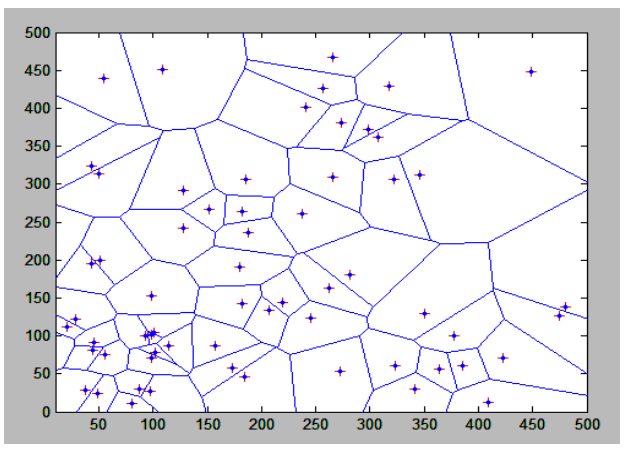

Figure 4: Clusters form

This figure 4 presents cluster formation. Here applied proposed algorithm i.e hybrid cluster approach. The combination of Energy Efficient Hierarchical Clustering and Modified low energy adaptive clustering hierarchy are using for joint clustering. 


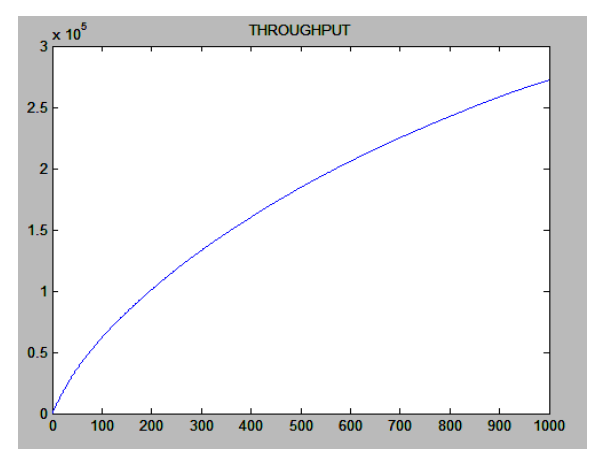

Figure 5: Data transmission rate or throughput

This figure 5 presents the data transmission rate or throughput rate. It is clear that the throughput is $275 \mathrm{Kbps}$.

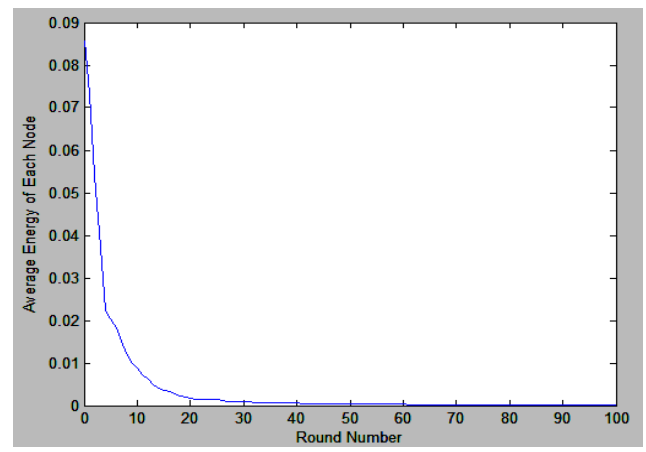

Figure 6: Average energy of each node

This figure 6 shows that the average energy of each nodes. Therefore $0.1 \mathrm{~J}$ to approx negligible energy take by each node.

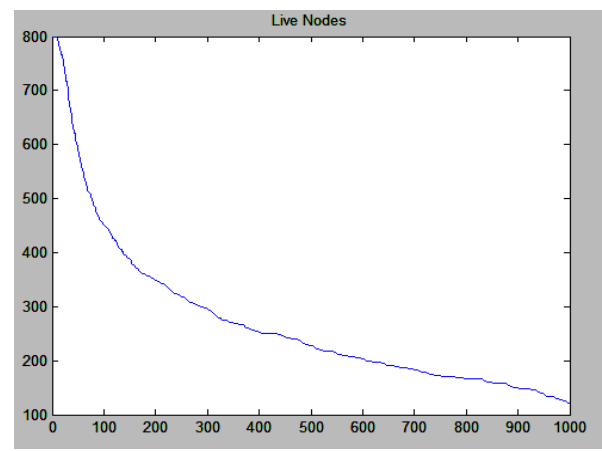

Figure 7: live nodes

This figure 7 shows that the total lives nodes during round of simulation. Therefore total 200 nodes live in 1000 rounds. 


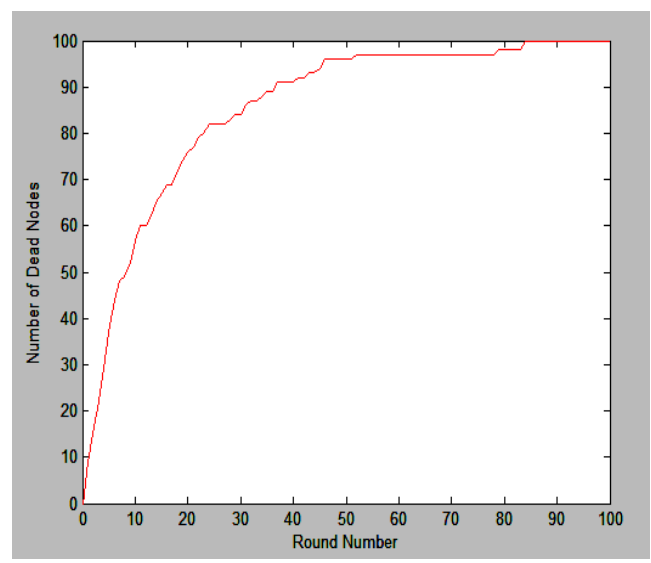

Figure 8: Dead nodes

This figure 8 shows that the total dead nodes count. During simulation nodes loss energy and it become dead during execution time. Therefore aprox 100 nodes dead.

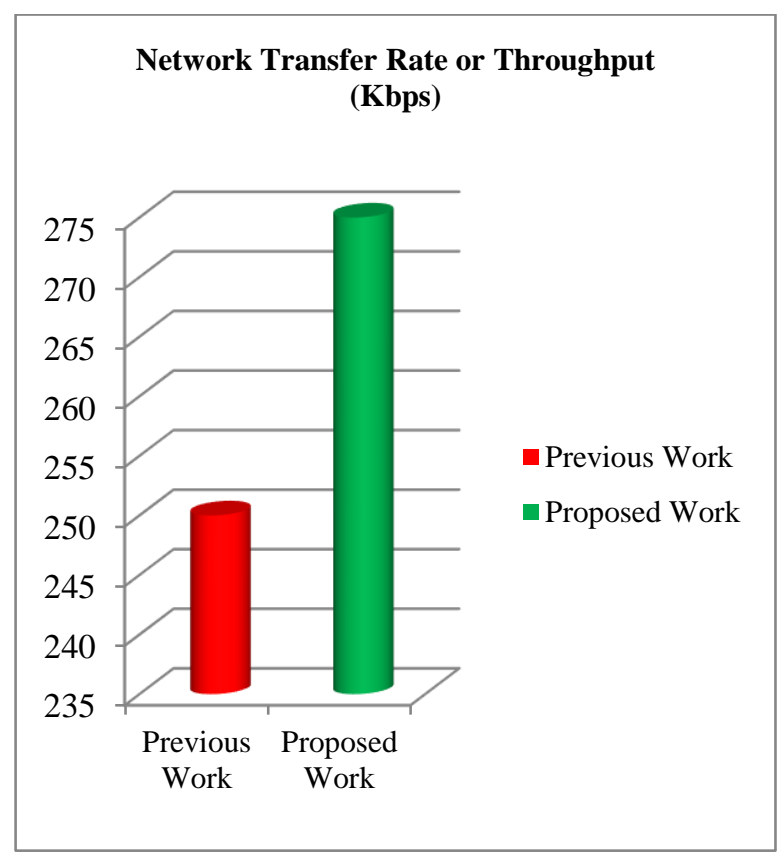

Figure 9: Comparison of Network transfer rate

Table 1: Comparison of proposed work result with previous work

\begin{tabular}{|c|l|l|l|}
\hline $\begin{array}{l}\text { Sr } \\
\text { No. }\end{array}$ & Parameters & $\begin{array}{l}\text { Previous } \\
\text { Work }\end{array}$ & $\begin{array}{l}\text { Proposed } \\
\text { Work }\end{array}$ \\
\hline 1 & $\begin{array}{l}\text { Simulation } \\
\text { area }\end{array}$ & $\begin{array}{l}500 \mathrm{~m} \\
\text { X500m }\end{array}$ & $\begin{array}{l}500 \mathrm{~m} \\
\text { X500m }\end{array}$ \\
\hline 2 & Total nodes & $\begin{array}{l}200 \text { to } \\
800\end{array}$ & $\begin{array}{l}200 \text { to } \\
800\end{array}$ \\
\hline 3 & $\begin{array}{l}\text { Methodol- } \\
\text { ogy }\end{array}$ & $\begin{array}{l}\text { Novel } \\
\text { Approach }\end{array}$ & $\begin{array}{l}\text { Hybrid } \\
\text { Cluster }\end{array}$ \\
\hline 4 & $\begin{array}{l}\text { Network } \\
\text { transfer rate }\end{array}$ & 250 Kbps & 275 Kbps \\
\hline
\end{tabular}




\begin{tabular}{|c|l|l|l|}
\hline & $\begin{array}{l}\text { (Through- } \\
\text { put) }\end{array}$ & & \\
\hline 5 & Data size & 200 byte & 400 byte \\
\hline 6 & $\begin{array}{l}\text { Node En- } \\
\text { ergy con- } \\
\text { sumed }\end{array}$ & $5 \mathrm{~nJ}$ & $1 \mathrm{~nJ}$ \\
\hline
\end{tabular}

\section{Conclusion}

In this paper, we have proposed a HCR system for node performance improvement. This paper proposed hybrid clustering protocol approach. It consider total simulation area 500X500 meter and nodes area taken 200, 800 during nodes simulation. Proposed method based on hybrid cluster while previous approach based on novel method. Network transfer rate or throughput is achieved by proposed method is $275 \mathrm{Kbps}$ while previous it is achieved $250 \mathrm{Kbps}$. Simulation time is also reduced upto $835 \mathrm{Sec}$. The overall energy consumption is $380 \mathrm{~J}$ while previous it is 1000J. Therefore it can be say that the proposed methodology approach gives significant better performance in wireless sensor netowrk than previous approach. The future advancement of the work can be more advancement can be done in the proposed method so that network lifetime by using different more approaches.

\section{REFERENCES}

1. S. Lata, S. Mehfuz, S. Urooj and F. Alrowais, "Fuzzy Clustering Algorithm for Enhancing Reliability and Network Lifetime of Wireless Sensor Networks," in IEEE Access, vol. 8, pp. 66013-66024, 2020.

2. M. A. Hossen and S. Yoo, "Q-Learning Based Multi-Objective Clustering Algorithm for Cognitive Radio Ad Hoc Networks," in IEEE Access, vol. 7, pp. 181959-181971, 2019, doi: 10.1109/ACCESS.2019.2959313.

3. X. He, X. Fu and Y. Yang, "Energy-Efficient Trajectory Planning Algorithm Based on Multi-Objective PSO for the Mobile Sink in Wireless Sensor Networks," in IEEE Access, vol. 7, pp. 176204176217, 2019, doi: 10.1109/ACCESS.2019.2957834.

4. W. He, "Energy-Saving Algorithm and Simulation of Wireless Sensor Networks Based on Clustering Routing Protocol," in IEEE Access, vol. 7, pp. 172505-172514, 2019, doi: 10.1109/ACCESS.2019.2956068.

5. W. Osamy, A. M. Khedr, A. Aziz and A. A. El-Sawy, "Cluster-Tree Routing Based Entropy Scheme for Data Gathering in Wireless Sensor Networks," in IEEE Access, vol. 6, pp. 77372-77387, 2018, doi: 10.1109/ACCESS.2018.2882639.

6. S. Phoemphon, C. So-In and N. Leelathakul, "Optimized Hop Angle Relativity for DV-Hop Localization in Wireless Sensor Networks," in IEEE Access, vol. 6, pp. 78149-78172, 2018, doi: 10.1109/ACCESS.2018.2884837.

7. S. Si, J. Wang, C. Yu and H. Zhao, "Energy-Efficient and Fault-Tolerant Evolution Models Based on Link Prediction for Large-Scale Wireless Sensor Networks," in IEEE Access, vol. 6, pp. 7334173356, 2018, doi: 10.1109/ACCESS.2018.2882389.

8. H. Wu, F. Yao, Y. Chen, Y. Liu and T. Liang, "Cluster-Based Energy Efficient Collaborative Spectrum Sensing for Cognitive Sensor Network," in IEEE Communications Letters, vol. 21, no. 12, pp. 2722-2725, Dec. 2017, doi: 10.1109/LCOMM.2017.2758376.

9. K. Rajeswari and S. Neduncheliyan, "Genetic algorithm based fault tolerant clustering in wireless sensor network," in IET Communications, vol. 11, no. 12, pp. 1927-1932, 248 2017, doi: 10.1049/iet-com.2016.1074. 
10. L. Sivagami and J. Martin Leo Manickam, "Cluster-Based MAC Protocol for Collision Avoidance and TDMA Scheduling in Underwater Wireless Sensor Networks," in The Computer Journal, vol. 59, no. 10, pp. 1527-1535, Oct. 2016, doi: 10.1093/comjnl/bxw049.

11. A. Ray and D. De, "Energy efficient clustering protocol based on K-means (EECPK-means)-midpoint algorithm for enhanced network lifetime in wireless sensor network," in IET Wireless Sensor Systems, vol. 6, no. 6, pp. 181-191, 12 2016, doi: 10.1049/iet-wss.2015.0087.

12. P. T. A. Quang and D. Kim, "Clustering algorithm of hierarchical structures in large-scale wireless sensor and actuator networks," in Journal of Communications and Networks, vol. 17, no. 5, pp. 473481, Oct. 2015, doi: 10.1109/JCN.2015.000085. 